\title{
Early-Stage Waves in the Retinal Network Emerge Close to a Critical State Transition between Local and Global Functional Connectivity
}

\author{
Matthias H. Hennig, ${ }^{1}$ Christopher Adams, ${ }^{2}$ David Willshaw, ${ }^{1}$ and Evelyne Sernagor ${ }^{2}$ \\ ${ }^{1}$ Institute for Adaptive and Neural Computation, School of Informatics, University of Edinburgh, Edinburgh EH8 9AB, United Kingdom, and ${ }^{2}$ Institute of \\ Neuroscience, Medical Sciences, University of Newcastle upon Tyne, Newcastle upon Tyne NE2 4HH, United Kingdom
}

\begin{abstract}
A novel, biophysically realistic model for early-stage, acetylcholine-mediated retinal waves is presented. In this model, neural excitability is regulated through a slow after-hyperpolarization (sAHP) operating on two different temporal scales. As a result, the simulated network exhibits competition between a desynchronizing effect of spontaneous, cell-intrinsic bursts, and the synchronizing effect of synaptic transmission during retinal waves. Cell-intrinsic bursts decouple the retinal network through activation of the sAHP current, and we show that the network is capable of operating at a transition point between purely local and global functional connectedness, which corresponds to a percolation phase transition. Multielectrode array recordings show that, at this point, the properties of retinal waves are reliably predicted by the model. These results indicate that early spontaneous activity in the developing retina is regulated according to a very specific principle, which maximizes randomness and variability in the resulting activity patterns.
\end{abstract}

Key words: retinal development; retinal waves; computational model; percolation; phase transition; correlated activity

\section{Introduction}

Retinal waves are a well studied example of spontaneous, patterned activity during CNS development and have been characterized in many vertebrate species (Wong, 1999). They appear before the retina is capable of responding to light and are observed as rhythmic bursts of action potentials in retinal ganglion cells (RGCs) that propagate in wave-like events across the surface of the retina. An involvement of retinal waves in visual system development has long been hypothesized, and recent studies demonstrate that manipulating wave activity can indeed affect the maturation of the projections of RGC axons (Firth et al., 2005) and the development of the retinal circuitry (Sernagor and Grzywacz, 1996; Sernagor et al., 2001; Mehta and Sernagor, 2006). It is also increasingly clear that in some cases specific properties of retinal waves are required for normal development (Muir-Robinson et al., 2002; McLaughlin et al., 2003; Cang et al., 2008; Shah and Crair, 2008; Sun et al., 2008). This suggests that

Received 0ct. 9, 2008; revised Dec. 19, 2008; accepted Dec. 22, 2008.

This work was supported by the Edinburgh Compute and Data Facility [partially supported by the eDIKT (eScience Data, Information and Knowledge Transformation) initiative]. We thank members of the CARMEN (Code Analysis, Repository \& Modelling for E-Neuroscience) consortium for providing infrastructure for data sharing. M.H.H. was supported by a United Kingdom Medical Research Council Fellowship (G0501327), and E.S. was supported by grants from the Engineering and Physical Sciences Research Council (EP/E002331/1) and the Centre of Excellence for Life Sciences Limited (OneNorthEast). We thank Drs J. Cortes, S. Eglen, M. Herrmann, M. Muñoz, and M. van Rossum for discussions.

Correspondence should be addressed to Matthias H. Hennig, Institute for Adaptive and Neural Computation, School of Informatics, University of Edinburgh, 10 Crichton Street, Edinburgh EH8 9AB, UK. E-mail: m.hennig@ed.ac.uk.

DOI:10.1523/JNEUROSCI.4880-08.2009

Copyright $\odot 2009$ Society for Neuroscience $\quad$ 0270-6474/09/291077-10\$15.00/0 the adequate regulation of spontaneous retinal activity is an important prerequisite for normal visual system development.

Important questions remain unresolved in terms of understanding the physiological processes underlying the typical spatiotemporal properties of retinal waves and how they are maintained and regulated during development. Although some wave features, such as their frequency of occurrence or propagation velocity, are highly variable across species, there are also important commonalities. In particular, in all species investigated so far, retinal waves display a high degree of randomness. Successive retinal waves occur at variable intervals, and exhibit a high degree of variability and randomness in terms of initiation point, size and trajectory (Feller et al., 1997; Stellwagen et al., 1999). Similar forms of spontaneous activity have also been found in other developing neural structures including the hippocampus and spinal cord (Feller, 1999; O’Donovan, 1999).

Here, by combining biophysical modeling and multielectrode array (MEA) recordings, we show that retinal waves arise at a very specific network state which can be interpreted in terms of a percolation model (Essam, 1980), a classical model in statistical mechanics. In particular, we demonstrate that a slow afterhyperpolarization (sAHP) current that has recently been characterized in starburst amacrine cells (SACs) (Zheng et al., 2006) acts on two different time scales, providing a mechanism to desynchronize neurons and dynamically regulate the network connectedness. A mean field approximation shows that this network can undergo a percolation phase transition, separating the states of purely local and global functional connectedness. A comparison of simulated activity with experimental MEA recordings shows that retinal waves emerge close to this tran- 
sition point, which is manifested as power-law distributions of wave sizes and durations.

\section{Materials and Methods}

Computational model. The model implements a regular, twodimensional, hexagonal lattice of starburst amacrine cells. Starburst amacrine cells were modeled using the membrane equation, with a membrane capacity $C=160 \mathrm{pF}$, resistance $R=180 \mathrm{M} \Omega$ and resting potential $V_{\mathrm{r}}=-65 \mathrm{mV}$. Synaptic inputs and membrane channels have conductances $g_{\mathrm{i}}(t)$ and associated reversal potentials $E_{\mathrm{r}}^{\mathrm{i}}$. A fast voltagedependent calcium current was modeled using an adiabatic approximation of its fast dynamics (Morris and Lecar, 1981):

$$
g_{\mathrm{Ca}}(t)=0.5 g_{\mathrm{m}}^{\mathrm{Ca}}\left(1+\tanh \frac{V(t)-V_{\mathrm{HCa}}}{2 V_{\mathrm{SCa}}}\right) .
$$

The half-activation point was set to $V_{\mathrm{Hca}}=-20 \mathrm{mV}$, the slope $V_{\mathrm{SCa}}=10$ $\mathrm{mV}$, the peak conductance $g_{\mathrm{m}}^{\mathrm{Ca}}=10 \mathrm{nS}$, and the reversal potential $E_{\mathrm{r}}^{\mathrm{Ca}}=$ $50 \mathrm{mV}$. An sAHP current was modeled as a calcium-dependent potassium conductance with four gating particles $r(t)$, similar to $I_{\text {sAHP }}$ in cortical neurons (Abel et al., 2004). This current depends on the intracellular calcium concentration $[\mathrm{Ca}(t)]$, and a second, slower calcium-dependent process $s(t)$ :

$$
\begin{gathered}
g_{\mathrm{sAHP}}(t)=g_{\mathrm{m}}^{\mathrm{sAHP}} r(t)^{4}, \\
\tau_{\mathrm{r}} \frac{d r(t)}{d t}=(\alpha[\mathrm{Ca}(t)]+s(t))(1-r(t))-r(t), \\
\tau_{\mathrm{s}} \frac{d s(t)}{d t}=\beta \frac{[\mathrm{Ca}(t)]^{4}}{\gamma+[\mathrm{Ca}(t)]^{4}}-s(t) .
\end{gathered}
$$

The peak conductance was $g_{\mathrm{m}}^{\mathrm{sAHP}}=30 \mathrm{nS}$, the reversal potential $E_{\mathrm{r}}^{\mathrm{sAHP}}=$ $-90 \mathrm{mV}$, the activation constant $\alpha=2400$, and the decay time $\tau_{\mathrm{r}}=5 \mathrm{~s}$. A cooperative activation of the slow calcium-dependent process was assumed, with an activation constant $\beta=30$, Michaelis constant $\gamma=10^{-8}$ and decay time $\tau_{\mathrm{s}}=50 \mathrm{~s}$. The calcium concentration was calculated as follows:

$$
\tau_{\mathrm{C} a} \frac{d[\mathrm{Ca}(t)]}{d t}=-[\mathrm{Ca}(t)]+\delta I_{\mathrm{Ca}}(t),
$$

with scaling factor $\delta=10^{8}$ and decay time $\tau_{\mathrm{Ca}}=50 \mathrm{~ms}$. Neurons have an intrinsic source of noise, which was implemented as filtered shot noise:

$$
\tau_{\mathrm{n}} \frac{d I_{\mathrm{n}}}{d t}=\alpha P(t, \lambda(V))\left(V(t)-E_{\mathrm{r}}^{\mathrm{n}}\right)-I_{\mathrm{n}},
$$

where $P(t, r(V))$ is a Poisson process with a voltage-dependent rate $r(V)$, event size $\alpha=200 \mathrm{pS}$ and reversal potential $E_{\mathrm{r}}^{\mathrm{n}}=50 \mathrm{mV}$. The source of cell-intrinsic noise in SACs is not known at present, and may be the result of stochastic ion channel gating, so a voltage-dependent rate-modulated Poisson process was used with $\lambda(t)=k_{0} p(V)(1-p(V))$, where $p(V)$ was the calcium channel activation function in Equation 1, and $k_{0}=1400 / \mathrm{s}$. These activation variables were chosen as simulations suggest that low calcium channel densities in small, distal SAC dendrites can induce spontaneous firing in SACs (M. H. Hennig, unpublished data). Simulations have, however, also shown that the same results are obtained with other noise models, such as an unmodulated Poisson process (data not shown).

SACs receive excitatory input from all neighboring cells $j$ within in a circular region of a radius of three cells with a Gaussian distancedependent synaptic weight with $\mathrm{SD} \sigma_{\mathrm{w}}=1$. The threshold for transmitter release was $V_{\mathrm{T}}=-60 \mathrm{mV}$, and synaptic currents were low-pass filtered with a time constant $\tau_{\text {syn }}=0.2 \mathrm{~s}$ to account for the slow cholinergic transmission in immature SACs (Zheng et al., 2004). The total synaptic conductance is then $g_{\text {syn }}=g_{\text {syn }}^{\max } \sum_{j} g_{\text {syn }}^{j}$ with $g_{\text {syn }}^{\max }=320 \mathrm{nS}$. A forward Euler scheme was used for integration, with a time step of $1 \mathrm{~ms}$. Unless stated otherwise, statistical measures were obtained from $4000 \mathrm{~s}$ simulated activity on a lattice of $56 \times 56$ SACs, where the central $50 \times 50$ neurons were used for analysis to reduce boundary effects. The simula- tion software was implemented in $\mathrm{C}++$. The source code is available upon request.

MEA recordings. This study was done using C57b1/6 neonatal mice and turtle embryos from the species Pseudemys Scripta Elegans. All animal procedures were conducted under the United Kingdom Home Office, Animals (Scientific procedures) Act 1986. Mouse pups were killed by cervical dislocation and enucleated before retinal isolation. Turtle embryonic ages were determined according to specific staging criteria (Yntema, 1968). The embryos were anesthetized by hypothermia, decapitated and enucleated before retinal isolation. The isolated retina was then transferred to the experimental chamber and placed, RGC layer facing down, onto MEAs consisting of 60 titanium nitride electrodes (30 $\mu \mathrm{m}$ diameter, $200 \mu \mathrm{m}$ spacing) arranged in an $8 \times 8$ grid on indium tin oxide substrate (Multi Channel Systems). Better coupling between the tissue and the electrodes was achieved by placing a small piece of polyester membrane filter ( $5 \mu \mathrm{m}$ pores) (Sterlitech) on the retina followed by a slice anchor holder (Warner Instruments). For mice, the retina was kept at $32^{\circ} \mathrm{C}$ and continuously perfused $(2-5 \mathrm{ml} / \mathrm{min}$ ) with artificial CSF containing the following (in mM): $118 \mathrm{NaCl}, 25 \mathrm{NaHCO}_{3}, 1 \mathrm{NaH}_{2} \mathrm{PO}_{4}, 3 \mathrm{KCl}$, $1 \mathrm{MgCl}_{2}, 2 \mathrm{CaCl}_{2}$, and 10 glucose, equilibrated with $95 \% \mathrm{O}_{2}$ and $5 \% \mathrm{CO}_{2}$. For turtles, the retina was kept at $29^{\circ} \mathrm{C}$ and perfused with Ringer's solution containing the following (in $\mathrm{mm}$ ): $96.5 \mathrm{NaCl}, 2.6 \mathrm{KCl}, 2 \mathrm{MgCl}_{2}, 31.5$ $\mathrm{NaHCO}_{3}, 10$ glucose, $10 \mathrm{HEPES}$ and $4 \mathrm{CaCl}_{2}$, equilibrated with $95 \% \mathrm{O}_{2}$ and $5 \% \mathrm{CO}_{2}$. Signals were amplified (gain 1200) and acquired using a 128-channel analog to digital converter (Multi Channel Systems MC_Card). Signals were digitized at $25 \mathrm{kHz}$ and acquired without filtering using the software MC_Rack (Multi Channel Systems). The time of occurrence of spontaneous spikes was threshold-detected with MC_Rack (the typical threshold was at signal amplitude that is $3 \times$ below the baseline noise). Hence, the firing rate on each electrode reflects the overall activity level generated by all RGCs on that same electrode (typically $4-5$ cells). Using the software MC_Data Tools (Multi Channel Systems), the times of spike occurrence were converted into text files for further analysis. Retinal waves were then detected using custom software written in Matlab (Version 7.21; Mathworks).

Analysis of MEA recordings. Spike trains of MEA recordings of retinal waves typically show occasional bursts of spikes at high rates, which are embedded in low-frequency background activity. For a precise and reliable burst detection, first the ranks $R(t)$ for the interspike intervals and the probability distribution $P\left(C_{\mathrm{s}}\right)$ for the spike count in a fixed time window were obtained (mouse, $1 \mathrm{~s}$; turtle, $4-6 \mathrm{~s}$; these values reflect the typical burst duration). Then, each spike train was analyzed spike by spike, and bursts were detected using a combination of two threshold criteria based on the mean firing rate on each electrode. Specifically, the onset of a burst was defined as an event where $R(t)<\theta_{\mathrm{r}}$, where the rank threshold $\theta_{\mathrm{r}}$ was manually adjusted (typical value for mouse data were $\theta_{\mathrm{r}}$ $=0.2$ and for turtle data $\theta_{\mathrm{r}}=0.1$ ), and where the spike count $C_{\mathrm{s}}$ in the fixed window beginning at the current spike exceeded a threshold. The threshold was taken as the value of $C_{\mathrm{s}}$ where $P\left(C_{\mathrm{s}}\right)=\theta_{\mathrm{s}}$ (mouse, $\theta_{\mathrm{s}}=$ 0.05 ; turtle, $\theta_{s}=0.3$ ), and calculated individually for each electrode. The end of a burst was determined as the time of the spike where the spike count first dropped below half of the onset threshold. This method was insensitive to the large differences in average and peak firing rates between different electrodes.

Waves were detected as temporally overlapping groups of bursts. The neighborhood relationship between electrodes was not taken into account, as occasionally multiple waves can be initiated at the same time on the area covered by the MEA (compare Fig. $4 A$ ). To avoid the overlap of temporally separated waves, a maximum duration was imposed on bursts ( $2.5 \mathrm{~s}$ or longer). This value was chosen because activity propagation between neighboring electrodes (with a distance of $200 \mu \mathrm{m}$, or 280 $\mu \mathrm{m}$ for the diagonal) is expected to take $2 \mathrm{~s}$ or less, since typical wave propagation velocities are $>100 \mu \mathrm{m} / \mathrm{s}$ (Wong et al., 1993; Feller et al., 1997). Visual inspection confirmed that this method was suitable for detection of coherent wave events, while shorter maximal burst durations could lead to splitting of waves. Not imposing a maximal duration had only minor effects for the mouse data, but was necessary for the turtle recordings, where bursts often lasted $10 \mathrm{~s}$ or longer.

Estimation of power-law exponents. To obtain reliable estimates of the 
parameters of the power-law distributions of wave sizes and lifetimes, and to test the quality of these fits, we used the methods outlined in detail by Clauset et al. (2007). In the following, we provide a brief summary of the way these techniques were applied here. A power-law probability distribution has the form

$$
p(x)=C x^{-\alpha},
$$

with $x \geq x_{\mathrm{m}}>0$ for all $x$ and $\alpha>1 . x_{\mathrm{m}}$ is the lower bound for the power-law distribution and $C$ the normalization constant. For realvalued $x$, we have $C=(\alpha-1) x_{\mathrm{m}}^{\alpha-1}$, and if $x$ is discrete valued, $C=\zeta(\alpha$, $\left.x_{\mathrm{m}}\right)^{-1}$, where $\zeta\left(\alpha, x_{\mathrm{m}}\right)$ is the generalized zeta function.

To estimate the power-law exponents for the distributions of retinal wave sizes and lifetimes, we used a combination of minimization of the Kolmogorov-Smirnov (KS) statistic to estimate the lower bound $x_{\mathrm{m}}$ and a maximum likelihood estimator (MLE) to estimate the exponent. For the discrete-valued wave-size distributions with $n$ data points, $\alpha$ was estimated by maximizing the log-likelihood function:

$$
L(\alpha)=-n \ln \zeta\left(\alpha, x_{\mathrm{m}}\right)-\alpha \sum_{i=1}^{n} \ln x_{i} .
$$

For the continuous lifetime distributions, a closed-form estimator for the exponent $\beta$ is given by the following:

$$
\beta=1+n\left(\sum_{i=1}^{n} \ln \frac{x_{\mathrm{i}}}{x_{\mathrm{m}}}\right) .
$$

Since the lower end of the distributions of the simulated and experimental data are affected by aliasing, we estimated power-law exponents and calculated the KS statistic for different values of $x_{\mathrm{m}}$ up to a prespecified limit $x_{\mathrm{m}}^{+}$. The value of $x_{\mathrm{m}}$ which minimized the KS statistic, and the corresponding exponent, were then taken as the best estimates. The fixed upper limits for the experimental data were $x_{\mathrm{m}}^{+}=6$ for the wave-size distributions and $x_{\mathrm{m}}^{+}=5 \mathrm{~s}$ for the lifetime distributions, to prevent spurious effects due to the small sample sizes.

The goodness-of-fit was estimated by comparing the KS statistic for the real data to that obtained from synthetic data sampled from powerlaw distributions with the same parameters. To this end, 2000 synthetic data sets, each with the same number of data points as in the original set, and sampled from a power-law distribution with the same parameters, were generated, and their parameters were estimated. Then, the KS statistic was calculated for each data set, and the $p$ value was determined as the fraction of times the KS statistic for the synthetic data sets exceeded that for the original data. For experimental data sets, the estimates for the exponents and $x_{\mathrm{m}}$ were obtained as specified above. To reduce the time needed to compute the $p$ values for the discrete simulated wave-size distributions, which typically contained $>1000$ events, a continuous approximation for the MLE was used:

$$
\alpha_{\text {sim }}=1+n\left(\sum_{i=1}^{n} \ln \frac{x_{\mathrm{i}}}{x_{\mathrm{m}}-1 / 2}\right) .
$$

\section{Results}

\section{Characterization of simulated SAC activity}

Retinal waves early in development depend on cholinergic synaptic transmission between SACs (Feller et al., 1996; Zhou and Zhao, 2000), spontaneous, calcium-channel mediated bursts in SACs, and a sAHP conductance which regulates their intrinsic excitability (Zheng et al., 2006). These mechanisms have been implemented with a recurrent network of conductance-based model neurons, which reproduce the physiological properties of immature SACs.

An isolated simulated SAC produces infrequent, spontaneous bursts caused by cell-intrinsic noise (Fig. $1 A$, black traces). Calcium influx during a burst activates the sAHP current, which induces a strong, long-lasting hyperpolarization preventing further spontaneous bursts. Experimental manipulations have shown that longer or more intensive depolarizations increase the sAHP duration (Zheng et al., 2006). This was modeled by combining direct, calcium-dependent current activation with an additional slow, presumably second-messenger mediated process. The relaxation time of the current then depends on the amount of calcium influx and is longer after stronger or more intensive depolarization. This is illustrated in Figure $1 A$, which compares the effects of brief current injections with that of spontaneous bursts. In either case, calcium influx activates the sAHP current, which leads to a temporary dampening in cellular excitability and suppression of subsequent spontaneous bursts. Increasing the current intensity leads to a stronger activation of the slow component of the sAHP current, which in turn results in a longer suppression of spontaneous bursts. Therefore, the average latency of the first spontaneous burst after a current injection increases monotonously with the current magnitude, until it saturates at a value determined by the decay time of the slow sAHP component (Fig. $1 B$ ).

This differential activation of the sAHP current becomes important when SACs are synaptically coupled. Spontaneous bursts can then occur in isolation (Fig. 1C, asterisks), and coincident spontaneous bursts in nearby neurons trigger propagating waves of activity (Fig. $1 C$, diamonds). During a wave, strong synaptic drive from multiple, simultaneously active SACs leads to a stronger depolarization than intrinsic bursts, which in turn causes a longer-lasting reduction in excitability of the participating SACs. Since waves uniformly reduce the excitability of participating neurons, wave boundaries are determined by recently active retinal areas, as shown in previous studies (Feller et al., 1996, 1997).

Intrinsic bursts also temporarily reduce the excitability of single SACs, which can prevent their participation in a subsequent wave (Fig. 1C, arrow). Since the sAHP current induced by intrinsic bursts decays faster than following waves, this results in competition between a desynchronizing effect of intrinsic bursting, and the synchronizing effect of synaptic transmission. This requires that synaptic connections between SACs are sufficiently weak, such that an isolated intrinsic burst cannot trigger a propagating wave (Fig. $1 C$, asterisks). Under these conditions, propagating activity patterns emerge, exhibiting a mixture of randomness and spatial coherence as in experimentally recorded retinal waves. Two examples of simulated waves are shown in Figure 2, $A$ and $B$, illustrating that a wave only recruits neurons that are sufficiently depolarized to generate bursting activity, but stops when it encounters neurons with a reduced excitability due to recent activity (supplemental Movie, available at www.jneurosci.org as supplemental material).

\section{Simulated retinal waves}

An important prerequisite for the appearance of the characteristic random, noncyclic activity patterns of retinal waves is therefore the coexistence of synchronizing, propagating activity and desynchronizing, intrinsic bursting in the network. This condition is met for a range of physiologically plausible parameter combinations in the model. To determine when the typical properties of retinal waves are best reproduced, we investigated the properties of simulated waves for different intensities of the cellintrinsic noise (parameter $k_{0}$ in Eq. 6 ; note that this parameter reflects the peak noise rate, and that the actual rate is typically much lower as it also depends on the membrane potential). Changing the noise intensity directly affects the rate of spontane- 
ous, intrinsic bursting, and is hence best suited to determine its role in network activity desynchronization.

The simulations show that a gradual increase in noise intensity causes a transition between two qualitatively different types of spatio-temporal network activity (see also supplemental Fig. S1, available at www. jneurosci.org as supplemental material). For weak noise, the network produces a mixture of large and small waves at a low frequency, and the distributions of wave sizes and lifetimes have a bimodal form (Fig. 2C-E, blue curves). This reflects strongly correlated network activity, as evident in the slow decay of the spatial correlations (Fig. $2 F$, left), and occurs because SACs produce few intrinsic bursts that hinder the propagation of activity. The low-wave frequency is due to the low probability of simultaneous bursting of nearby neurons, which is required for wave initiation.

Increasing the noise intensity leads to the appearance of more frequent waves of intermediate size and duration (Fig. 2C-E, red curves). At a certain noise intensity $\left(k_{0}\right.$ $\approx 1.4 \mathrm{kHz}$; medium noise), the wave-size distribution changes from a bimodal to a monotonously decreasing function, and follows a power law with an exponent of $\alpha=1.5$. Rescaling the lattice of SACs by combining multiple neurons, and declaring each group of neurons as active only when more than half of the neurons are active (majority rule) yields the same power-law distributions (supplemental Fig. S2, available at www.jneurosci.org as supplemental material), confirming that the activity indeed has scale-free properties. At the same time, the wave lifetime distribution takes the form of an exponentially truncated power law with an exponent of $\beta=2$, which is also preserved after rescaling. In this case, the stronger desynchronizing effect of the more frequent intrinsic bursting activity leads to a faster decay of the spatial correlations than at low noise intensities (Fig. $2 F$, middle).

At noise intensities above this point, waves become even more frequent, but the largest waves are no longer observed, and the wave-size and lifetime distributions now have the form of exponentially truncated power-law functions (Fig, $2 C-E$, green curves). The correlations now decay rapidly, consistent with strong desynchronization of the network activity through very frequent intrinsic bursting (Fig. $2 F$, right). These simulated waves strongly resemble the patchy, nonpropagating activity observed in more mature animals (Sernagor et al., 2003; Syed et al., 2004).

These observations show that this network undergoes a transition from a highly synchronized to a strongly desynchronized state, and produces activity patterns clearly different from oscillatory, phased-locked behavior, where more compact event size
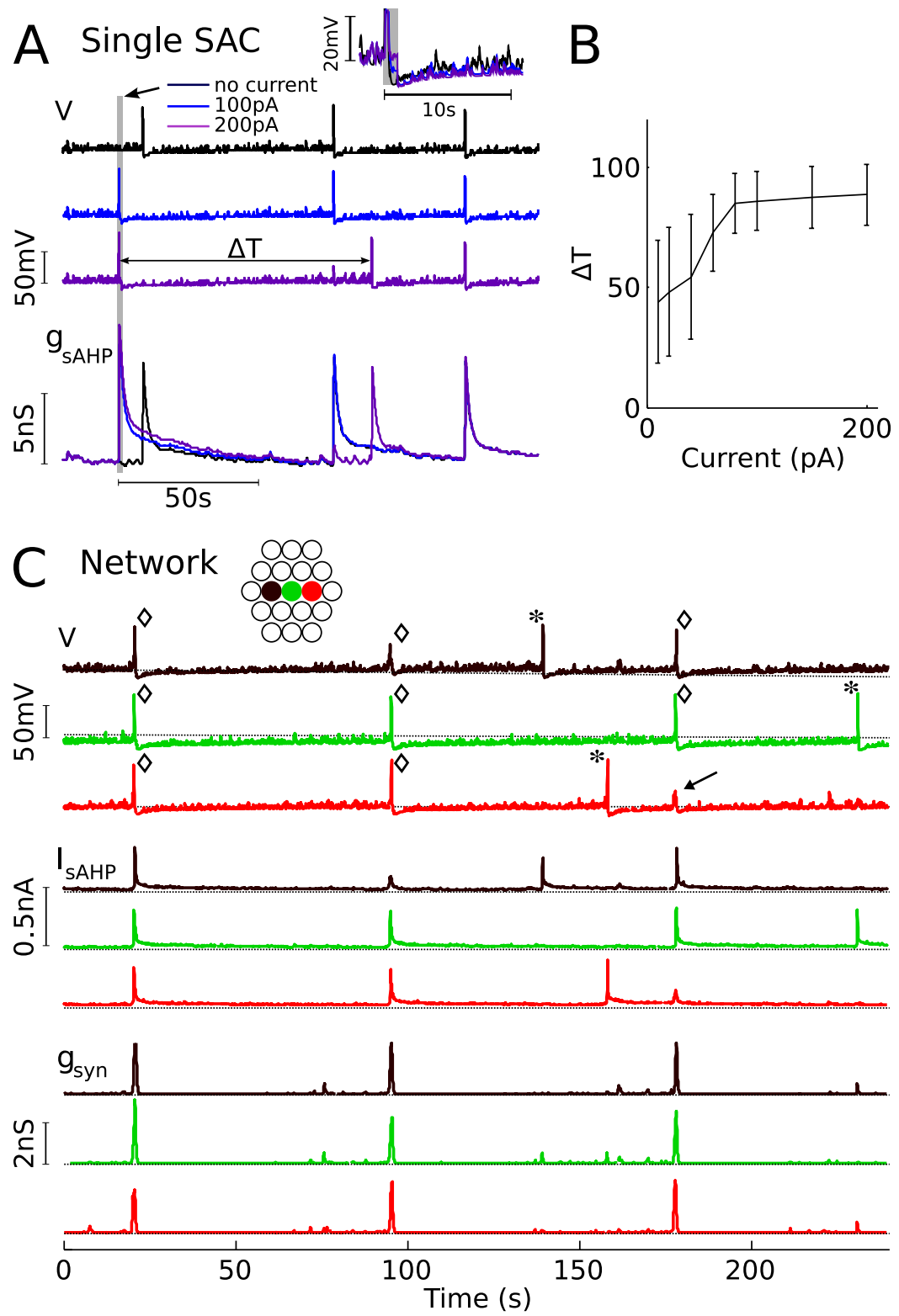

Figure 1. Activity-dependent activation of the SAHP conductance in simulated SACs. A, Membrane potential (top) and sAHP conductance (bottom) of an isolated SAC. The cell either produced noise-driven, intrinsic bursts (black traces), or depolarized in response to a current injection of $100 \mathrm{pA}$ (blue) or $200 \mathrm{pA}$ (purple) lasting $0.5 \mathrm{~s}$ (arrow). Increasingly stronger depolarizations during current injections cause a slower decay of the SAHP conductance than intrinsic bursts. The inset shows a comparison of a single intrinsic burst and of events evoked by the current injections. In all simulations, the same noise current was applied. $\boldsymbol{B}$ Average latency of the first spontaneous, intrinsic burst after different amounts of injected current $(0.5 \mathrm{~s}$; error bars indicate \pm 1 SD). C, Membrane potential (top), SAHP current (SAHP conductance multiplied by the driving force, middle) and synaptic conducintrinsic events; diamonds indicate activity during a wave. The arrow indicates a cell receiving strong synaptic input but not bursting in response due to reduced excitability after a previous intrinsic burst.

and interval distributions are expected. The observed noncyclic behavior also manifest itself through the random, unbiased distribution of wave initiation points (Fig. 2G), a central feature of retinal waves (Feller et al., 1996). Yet, subsequent wave trajectories are not entirely random (Fig. $2 H$ ) but consistent with a history-dependent regulation of the neural excitability (Feller et al., 1997).

\section{Simulated of pharmacological manipulations}

So far, the transition from the synchronized to the desynchronized network state has been characterized by varying the noise 

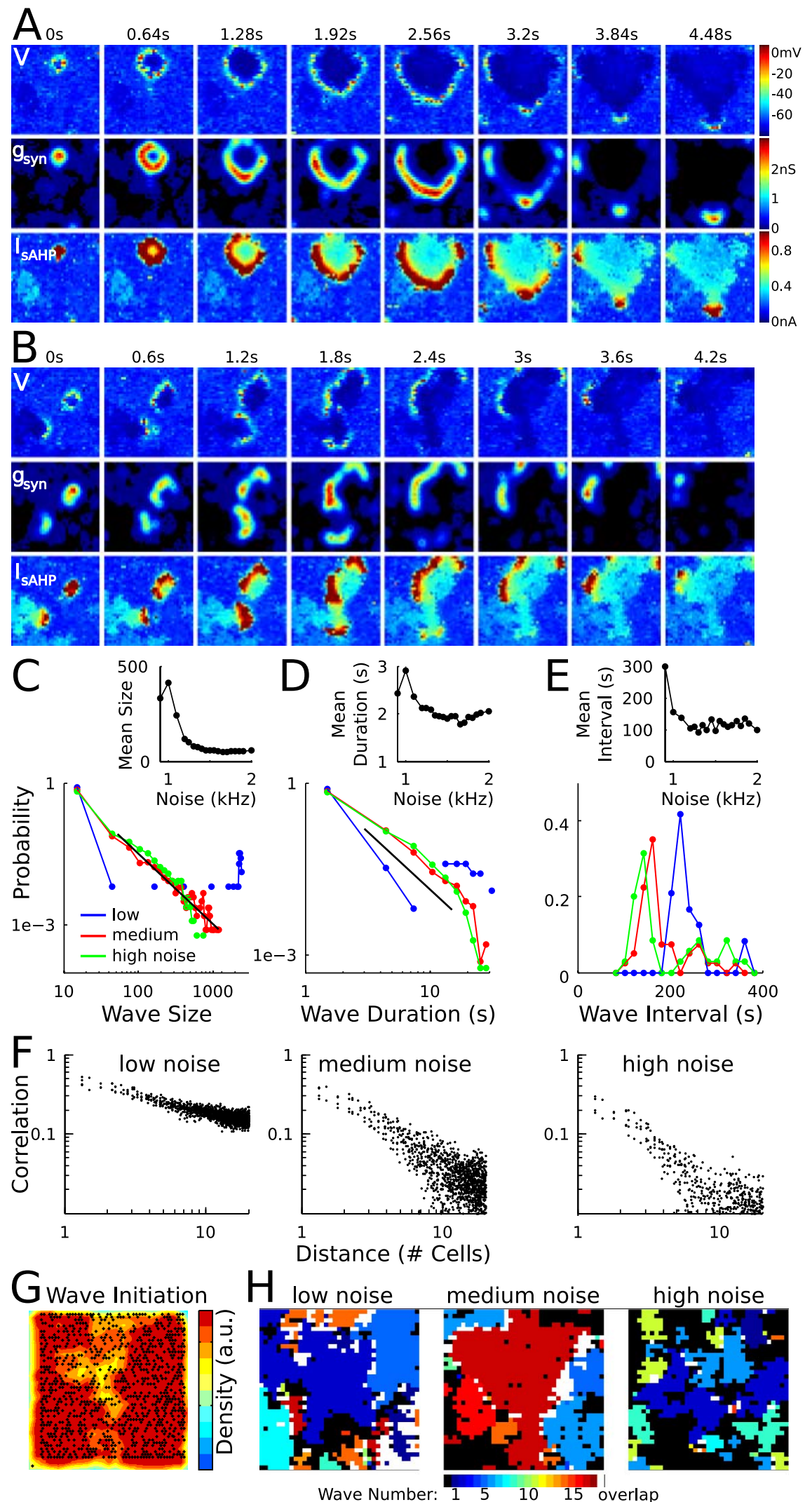

Figure 2. Characterization of simulated retinal waves. $\boldsymbol{A}, \boldsymbol{B}$, Two examples of a simulated retinal wave. Panels show the color-coded membrane potential (top), the synaptic conductance (middle), and the sAHP current (bottom). Color bars are the same in $\boldsymbol{A}$ and $\boldsymbol{B}$. $\boldsymbol{C}-\boldsymbol{E}$, Probability distributions and average values (insets) of the wave size (C), the wave duration $(\boldsymbol{D})$ and wave interval $(\boldsymbol{E})$ as a function of the cell-intrinsicnoise intensity (low, $k_{0}=1000 \mathrm{~Hz}$; medium, $k_{0}=1400 \mathrm{~Hz}$; high, $k_{0}=1800 \mathrm{~Hz}$ ). Lines show power-law functions with exponents $\alpha=-1.5(\boldsymbol{C})$ and $\beta=-2(\boldsymbol{D})$. $\boldsymbol{F}$, Pairwise correlations of the membrane potential at three different noise intensities. $\boldsymbol{G}$, Wave initiation points and their color-coded density. $\boldsymbol{H}$, Areas occupied by 20 successive waves at three different noise intensities. The relative time of each wave is color coded, and areas of overlap are shown in white. intensity, but the same behavior can also be observed by systematically changing other, experimentally more accessible, parameters. In particular, certain pharmacological manipulations can either increase or decrease synchronization, and hence induce transitions between the two regimens. To directly reproduce and interpret these effects with the model, we initially choose parameters such that power-law distributions are obtained to reproduce normal retinal waves (see below), and then simulated experimental manipulations by changing appropriate parameters. First, we investigate the effects of partially blocking cholinergic synaptic transmission, which has been shown to reduce wave frequency and spatial (Bansal et al., 2000; Sernagor et al., 2000, 2003). To simulate this, the peak synaptic conductance was uniformly reduced in the model. Consistent with the experimental results, this leads to a decrease in mean wave frequency and wave size as well as duration (Fig. 3A). These effects occur because more coincident bursts are required to trigger a wave when synapses are weak. This causes not only a decrease in wave frequency, but also a relative increase of the rate of intrinsic, desynchronizing bursts. As a result, the network enters a more desynchronized state, which leads to the disappearance of the largest waves (Fig. 3A, left). The opposite effect, an increase of wave size, duration and frequency, is observed when the synaptic strength is increased in the model. This mimics the experimentally observed increased frequency of calcium transients in the chick retina during blockade of ACh breakdown (Catsicas et al., 1998). However, a general elevation of the synaptic drive with nicotinic $\mathrm{ACh}$ receptor (nAChR) agonists abolishes wave activity (Zhou and Zhao, 2000), perhaps due to receptor desensitization, ion channel inactivation or permanent activation of potassium conductances.

Second, an increase of cAMP levels, which reduces the strength and affect the kinetics of the sAHP current in SACs (Zheng et al., 2006), has been shown to increase wave size and frequency (Stellwagen et al., 1999). This could be reproduced by reducing either the SAHP peak conductance (Fig. $3 B$ ), or the activation variable of the slow activation component of the sAHP current (Fig. 3C). Both manipulations cause the cellular excitability to be restored more quickly after activity, and lead to more frequent waves. Additionally, the desynchronizing effect of intrinsic bursts, mediated by the sAHP current, is reduced. This increase the relative fre- 
quency of the largest waves, and results in bimodal wave-size distributions as has been observed in calcium-imaging experiments (Stellwagen et al., 1999). Enhancing the sAHP current or the slow activation component, however, causes the opposite effect, with a decrease in average wave size, duration and frequency. This manipulation has not been tested experimentally so far.

Collectively, these results show that qualitatively different manipulations of synaptic efficacy, cellular excitability or intrinsic noise intensity (compare Fig. $2 C-E$ ) all have similar effects on the synchronization of the activity of the network. Each of these variables can be used to induce transitions between the synchronized and desynchronized network state.

\section{Analysis of MEA recordings}

Our results raise the question which of these two network states can best reproduce retinal waves. Calcium-imaging studies indicate that wave-size distributions are decreasing, highly skewed functions (Feller et al., 1997; Stellwagen et al., 1999; Bansal et al., 2000), similar to the power-law distributions predicted by the model at the transition point between the synchronized and desynchronized state. To test this experimentally, MEA recordings from mouse and turtle retinas were performed at early developmental stages, where waves primarily depend on cholinergic synaptic transmission (Feller et al., 1996; Sernagor and Grzywacz, 1999; Sernagor et al., 2003; Syed et al., 2004). If a behavior similar to that found in imaging experiments is observed with MEA recordings covering larger retinal areas, this would provide strong support for powerlaw distributions since only a power-law function retains its shape during spatial rescaling. However, due to the spacing between electrodes, MEA recordings also undersample the RGC mosaic, which will affect the measurement of wave sizes. Simulations show that spatial undersampling distorts power-law distributions in related models (Priesemann et al., 2007; V. Priesemann, M. H. J. Munk, and M. Wibral, unpublished observations), and increases the estimated powerlaw exponents in our simulations (supplemental Fig. S3, available at www.jneurosci.org as supplemental material). Hence, we would generally expect to find more rapidly decaying functions than predicted by the model.

Waves were detected as single or multiple coincident bursts of spikes on multiple electrodes (Fig. 4A) (see Materials and Methods). The normalized histograms in Figure $4, B$ and $C$, shows that the wave-size and lifetime distributions can, with one exception (Fig. $4 E$ ), be fitted by power functions (see Materials and Methods for details on the fitting procedure). The estimated exponents are close to, but always larger that the predicted $\alpha=1.5$ for wave sizes and $\beta=2$ for lifetimes (ranges: $\alpha=1.55-1.93 ; \beta=2.32$
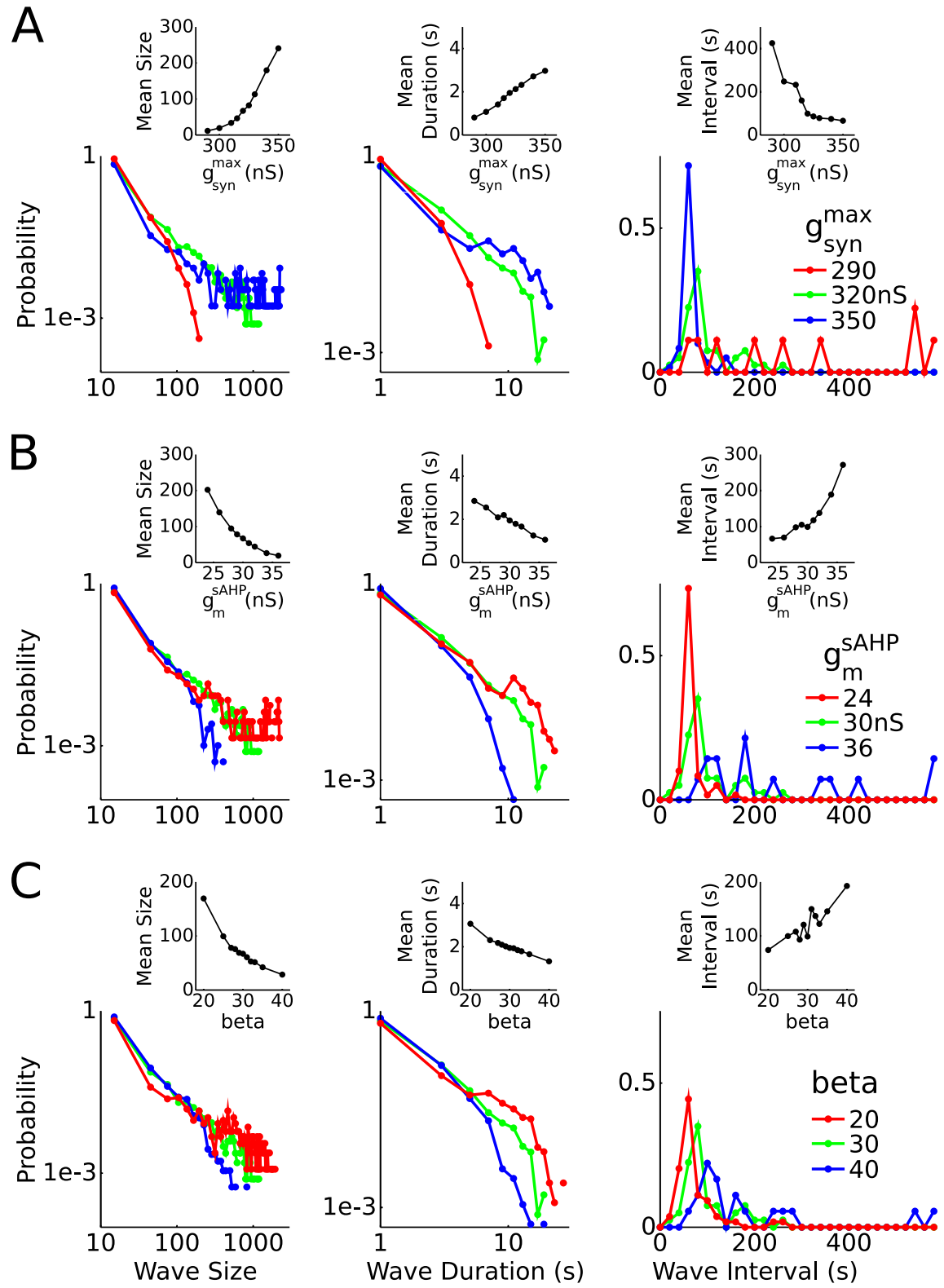

Figure 3. Summary of effects of changing different model parameters and their relation to experimental manipulations. Each part shows the distributions of wave sizes, durations and wave intervals and their mean values as a function of the manipulated wave duration histograms. $\boldsymbol{A}$, Effects of increasing or decreasing the peak synaptic conductance (default $\left.g_{\text {syn }}^{\max }=320 \mathrm{nS}\right) . \boldsymbol{B}, \boldsymbol{C}$, Effects of changing the sAHP peak conductance $\left(\boldsymbol{B}\right.$, default $\left.g_{\mathrm{m}}^{\text {sAHP }}=30 \mathrm{nS}\right)$, or its the slow activation constant $(\boldsymbol{C}$, default $\beta=30)$.

2.84). These deviations are expected for a moderate undersampling of the RGC mosaics (supplemental Fig. S3, available at www.jneurosci.org as supplemental material). We also estimated the goodness-of-fit by comparing the Kolmogorov-Smirnov statistic with that of synthetic data sets, which yields $p$ values to quantify the support for a power-law hypothesis (see Materials and Methods). However, simulations show that spatial undersampling also affects these $p$ values, in particular for the wave-size distributions (data not shown). Still, in one case, weak support for a power-law was found for the wave-size distribution (Fig. $4 D, \mathrm{P} 2$ mouse), and in four cases, this test supports the powerlaw hypothesis for the lifetime distributions (Fig. $4 B-D, G$ ). The analysis of two additional shorter dataset from postnatal day 5 (P5) mouse retinas produced similar results (data not illustrated; retina $1: 700$ s activity, 34 waves, $\alpha=1.78, p_{\alpha}=0.23, \beta=2.43, p_{\beta}$ 

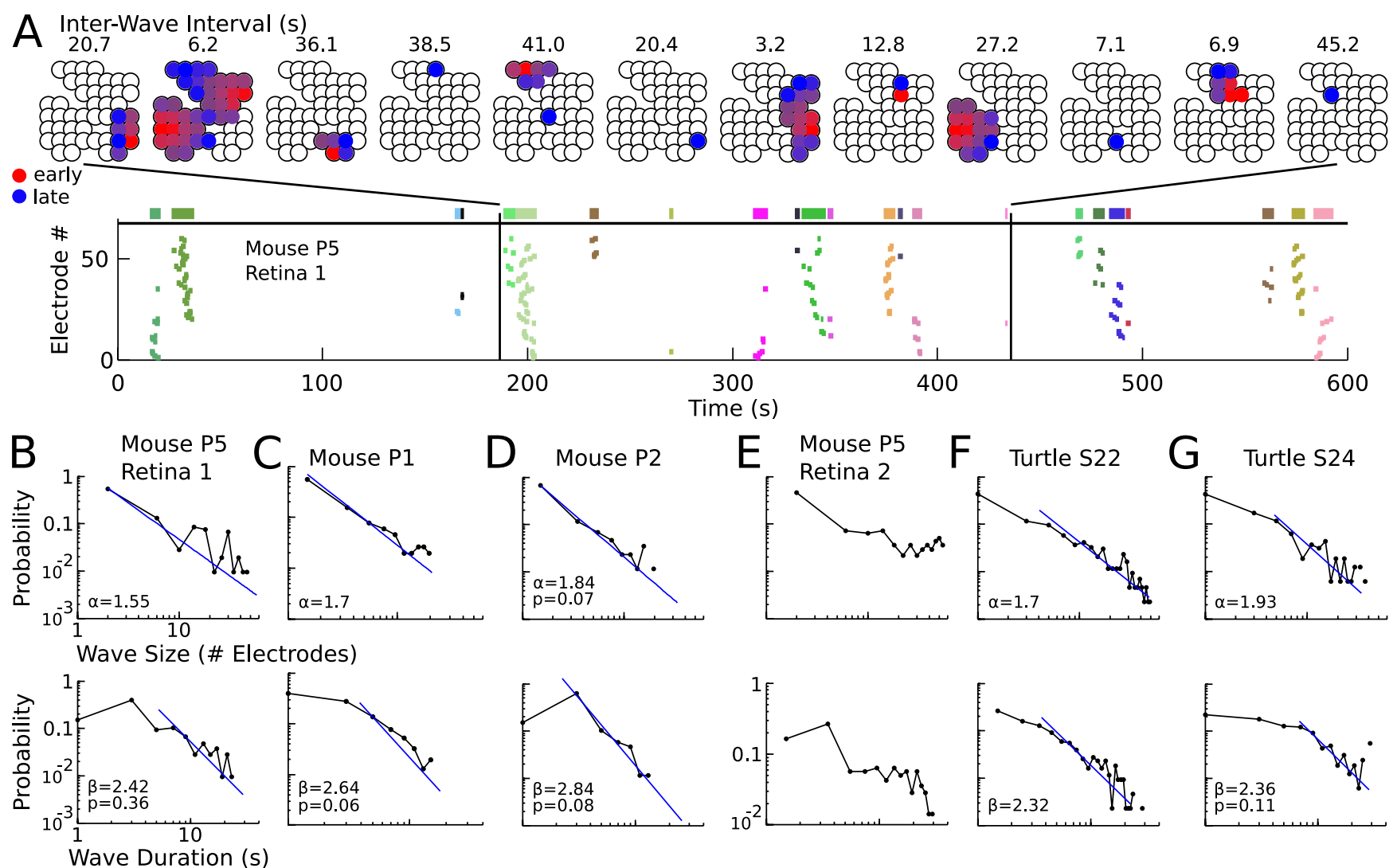

Figure 4. MEA recordings of early-stage retinal waves show power-law size and lifetime distributions. $A$, Spatio-temporal activity patterns of 12 individual waves (top, a color change from red to blue indicates the activation sequence) and bursting patterns (bottom; individual waves have different colors, and bursts assigned to the same wave have the same color) recorded from a mouse retina at day P5. $\boldsymbol{B}-\mathbf{G}$, Normalized histograms of wave sizes and lifetimes from six different retinas. Estimated exponents for the power laws are given in each graph, and $p$ values are given when $p>$ 0 (hence indicating support for the power law hypothesis). Blue lines show power-law functions with the estimated exponents. $\boldsymbol{B}$, Mouse retina at day $P 5,50$ min, as shown in $\boldsymbol{A}$ : 106 waves, mean interburst interval (IBI) $141 \pm 75$ s. C, Thirty minutes from a P1 mouse: 154 waves, mean IBI $107 \pm 63$ s. D, Sixteen minutes from a P2 mouse: 87 waves, mean IBI $112 \pm 61$ s. $E$, 0 ne and a half hours of activity from the second retina of the animal shown in $\boldsymbol{A}$ and $\boldsymbol{B}$ : 140 waves, mean IBI $131 \pm 73 \mathrm{~s}$. These distributions cannot be fitted with a power law but have a bimodal character. $\boldsymbol{F}, 0$ ne hour from an S22 turtle retina: 435 waves, mean IBI $51 \pm 27$ s. G, Thirty minutes from an S24 turtle retina: 163 waves, mean IBI $72 \pm 61 \mathrm{~s}$.

$=0.11$; retina $2: 750 \mathrm{~s}$ activity, 25 waves, $\alpha=1.73, p_{\alpha}=0.0, \beta=$ $2.25, p_{\beta}=0.08$; here, the small sample size may, however, introduced a bias). Together, these results are consistent with the model prediction that retinal waves exhibit statistical properties best described by power-law functions.

One retina from a P5 mouse did show bimodal size and lifetime distributions (Fig. $4 E$ ), and similar results were obtained in older animals (data not shown). Experimental observations suggest that around day P5 in mouse, GABAergic transmission begins to influence RGC spiking, increases spatial correlations and has subtle effects on wave spatial extent and frequency (Wang et al., 2007; E. Sernagor, unpublished data).GABA has a depolarizing effect at this developmental stage due to elevated $\left[\mathrm{Cl}^{-}\right]_{\mathrm{i}}$, so it is possible that maturation of the GABAergic drive causes an effective synaptic strengthening. In this case, the models predicts, as observed, bimodal distributions and increased spatial correlations (compare Fig. 3A).

\section{Percolation in the retinal network}

Power-law event size distributions are also called scale-free distributions, because their shape is unaffected by spatial scaling, such as a multiplication by a constant factor. Then, waves of all possible sizes are produced by the retina, ranging from very frequent waves involving just a few SACs to the very rare events of waves covering the whole retina. In other words, at this point, the properties of the desynchronized and the synchronized network states, characterized by small and large waves respectively, coexist. In a more abstract sense, this constitutes the typical signature of a continuous phase transition between an ordered and disordered network state. Similar phase transitions have been previously studied in related lattice models (Bak et al., 1987; Hopfield and Herz, 1995; Vespignani and Zapperi, 1998), and were suggested as an explanation for power-law event size distributions measured in cortical slices and cultures (Beggs and Plenz, 2003).

As outlined above, the sAHP-mediated desynchronization is a key mechanism to control the network state. It is activated by neural activity and temporally reduces the neural excitability. The size of a wave is, therefore, always determined by the number of excitable SACs available in the network, which in turn depends on the rate of waves and intrinsic bursting in SACs, and the size of previous waves. Therefore, on average, the central parameter that determines the wave properties is the density of excitable neurons. A system with these properties can be analyzed with methods from percolation theory, which was developed to describe the spreading of activity through a medium, where motion is solely determined by the stochastic properties of the medium (Essam, 1980). The corresponding mechanisms in our model are the propagation of neural activity through synapses, where the state of the neurons, excitable or refractory, dictates where activity can spread and where it cannot.

Importantly, percolation models display a continuous phase transition. When the density of excitable neurons is low, activity 
cannot spread far and waves are small. Increasing the density then also increases the mean wave size. At a particular density (the percolation threshold), the system undergoes a transition from a state of purely local connectedness to a state, where connections between neurons across the whole network can exist. This point is characterized by a diverging characteristic length scale, and as a result, the mean size of the clusters of connected neurons diverges. Then, propagating activity will assume power-law distributions. In particular, as observed here, the expected mean field exponents are $\alpha=1.5$ for wave sizes and $\beta=2$ for their lifetimes ( $M u n ̃ o z$ et al., 1999).

To describe our model in terms of a percolation model, we define three activity states of SACs: stable (S) for a refractory neurons, critical (C) for a nonrefractory neuron, and active (A) for a bursting neuron, with associated densities $\rho_{\mathrm{s}}$ and $\rho_{\mathrm{c}}$. The following state transitions are possible (Fig. 5A): Stable to critical with a probability $p(S: p \rightarrow C)$, and critical to active either with probability $f \rho_{c}$, if the neuron participates in a wave, or with probability $r$ in isolation, if it produces an intrinsic burst $\left(C: f \rho_{\mathrm{c}} \rightarrow A\right.$ and $\left.C: r \rightarrow A\right)$. Each firing event is always followed by a transition into the stable state, and firing events are brief and rare $(p \ll 1)$; hence, we can write $C: f \rho_{\mathrm{c}} \rightarrow S$ and $C: r \rightarrow S$. Assuming local homogeneity and ignoring higher orders in $\rho_{\mathrm{a}}$, the temporal evolution of $\rho_{\mathrm{c}}$ is then given by the following:

$$
\dot{\rho}_{\mathrm{c}}=p \rho_{\mathrm{s}}-\rho_{\mathrm{c}}\left(f \rho_{\mathrm{c}}+r\right),
$$

with $\rho_{\mathrm{s}}+\rho_{\mathrm{c}}=1$. Here, $p$ is not necessarily a fixed quantity and may depend on the previous activity. This allows for an estimation of the density of excitable neurons (the critical state density) through quantities that can be measured in simulations. To this end, we consider what happens after a burstor wave has occurred. Then, we obtain $\rho_{\mathrm{c}}^{+\mathrm{w}}=\rho_{\mathrm{c}}-f \rho_{\mathrm{c}}^{2}$ after a wave, and $\rho_{\mathrm{c}}^{+\mathrm{b}}=\rho_{\mathrm{c}}-r \rho_{\mathrm{c}}$ after an intrinsic burst. Assuming stationarity, i.e., $\rho_{\mathrm{c}}^{+\mathrm{w}} \approx \rho_{\mathrm{c}}^{+\mathrm{b}}$, yields the estimate $\rho_{\mathrm{c}}^{+} \approx \frac{r}{f}$. In other words, the mean density of critical states, after activity has occurred in the network, can be approximated as the ratio of the probabilities of isolated firing and firing during waves.

The estimated density of excitable neurons $\rho_{c}^{+}$as a function of the noise intensity is shown in Figure $5 B$. For weak noise, $\rho_{c}^{+}$is small, indicating that most cells simultaneously become refractory due to the predominantly very large waves. $\rho_{\mathrm{c}}^{+}$increases with increasing noise strength, consistent with a higher rate of intrinsic bursting and a more variable wave size. At the points where the wave-size distribution best approximates a power law with an exponent of $\alpha=1.5$ (Fig. $5 C$ ), the density of critical states is $\rho_{\mathrm{c}}^{+} \approx$ 0.7 . This value is very close to the threshold for site percolation on a hexagonal lattice $\left(p_{c} \approx 0.6970\right)$ (Suding and Ziff, 1999). Hence, wave-size power-law distributions are found at the point where our approximation predicts a percolation phase transition. When the noise intensity is further increased, $\rho_{c}^{+}$also increased and eventually exceeds unity. Then, the approximation is invalid be- cause the strong noise causes bursting in refractory neurons that cannot participate in waves.

In conclusion, these results show that the predicted and experimentally observed power-law statistics are consistent with a network state close to a percolation phase transition. Here, the dynamic regulation of the network excitability maintains a state at the boundary between a purely local and global connectedness of the SAC network.

\section{Discussion}

Returning to the issues raised at the beginning of this study, our results show that early-stage retinal waves indeed reflect a very specific network state characterized by highly variable spatiotemporal patterns, the absence of cyclic behavior, and power-law distributed wave sizes and lifetimes. Theoretical arguments show that these properties result from a dynamic regulation of the neural excitability, which maintain the SAC network close of a percolation phase transition. At this point, the network is at the transition point between a state of purely local and global functional connectedness. Local functional connectedness yields strongly desynchronized activity and small waves, and in the globally connected state, activity is highly synchronous with proportionally more large waves events. At the transition point, the neural activity simultaneously reflects both states, and therefore exhibits maximal variability with respect to wave sizes and durations. This is a defining feature of retinal waves (Feller et al., 1997), and we confirm the predicted power-law behavior with MEA recordings of retinal waves. Moreover, we demonstrate with the model how different pharmacological interventions can specifically shift the network away from this state.

\section{Developmental significance}

There are at least two reasons why such activity patterns may be relevance to neural development. The first is based on the consideration of what may constitute a stable state in the highly recurrent network in the immature retina and how it can be maintained and controlled by activity-dependent mechanisms. In particular, there may be two competing demands. On the one 
hand, the network has to maintain a certain level of activity, to ensure neural survival, to form and stabilize synapses, and to trigger cell-intrinsic activity-dependent developmental molecular pathways. Then, the synchronized, globally connected phase appears to be a suitable regimen. Yet, high synchrony also renders the network very unstable to small perturbations and leads to epileptiform activity. Therefore, it is at the same time necessary to limit the amount of synchrony. These two requirements are exactly balanced at the point of the phase transition. A similar effect has also been demonstrated recently in a randomly driven network model, where the stability and sensitivity to input was maximized at a transition from a quiescent regimen to a state with propagating, self-sustained activity (Ribeiro and Copelli, 2008). Since such behavior is also seen in cultured networks (see below), this may be a state which neural networks without an external drive generally adopt during development.

Second, it is now well established that spontaneous neural activity during development plays an important role in sculpting out the mature neural circuitry in various neural systems (Moody and Bosma, 2005). Here, we show that early-stage retinal waves exhibit maximal variability and containevents on all length scales due to their scale-free character. This randomness ensures that activity patterns are not biased toward a typical scale or sequence of events. This may be a general advantage while the retinal circuitry develops and retinal projections are established in visual centers of the brain. Indeed, the different correlation structure in early-stage waves in mice lacking the $\beta 2 \mathrm{AChR}$ subunit (Sun et al., 2008), for example, may cause a sustained lack of refinement of retinocollicular projections (McLaughlin et al., 2003) and prevent the development of eye-specific thalamic layers (MuirRobinson et al., 2002). Since our results suggest that early-stage retinal waves reflect a rather precisely regulated network state, it would be interesting to assess how sensitive higher visual system development is to modest changes of the spatio-temporal wave properties. In this context, it is interesting to note that the spatial frequency content in natural images also decays with a power law (Field, 1987), so retinal waves might present the visual system with an early opportunity to adapt to such patterns.

Clearly, this network state is not maintained during development. Changes toward a stronger emphasis on feedforward processing, as in the mature retina, might begin as soon as GABAergic synapses first appear, where we observed first deviations from the power-law behavior. At later stages, when glutamatergic transmission drives retinal waves, waves are small and patchy (Zhou and Zhao, 2000; Sernagor et al., 2003), which in our model would correspond to a state well below the percolation threshold for lateral activity propagation.

\section{Relations to other systems and previous models}

Power-law distributed event sizes and lifetimes with the same exponents $(\alpha=1.5$ and $\beta=2)$ were also found in cortical slices and cultures (Beggs and Plenz, 2003, 2004), and recently in the developing cortex in vivo (Gireesh and Plenz, 2008). These studies have proposed that this phenomenon could be explained in terms of a branching process, where a critical state exists when each spike in one neuron causes on average exactly one spike in another neuron. A critical branching process predicts power-law event size and lifetime distributions with the observed exponents, and is directly related to a large class of models that display the same type of phase transition (Zapperi et al., 1995). In models of neural networks, the same behavior has been observed in slowly driven recurrent network with nonleaky or nonrefractory neurons (Hopfield and Herz, 1995; Eurich et al., 2002; Buice and
Cowan, 2007), but theoretical work also shows that it may be difficult to reconcile this behavior with the biophysical properties of neurons (Dickman et al., 2000) (but see Levina et al., 2007). Here, we have identified a biologically plausible model that reproduces these properties. It is worth noting that the models mentioned above are generally different to the one presented here. Mathematical analysis has shown that the lattice all belong to the class of directed percolation models, where activity propagates forward in time (Vespignani and Zapperi, 1998; Buice and Cowan, 2007). In the present model, in contrast, the refractory mechanism prevents spreading of activity into previously active regions, and it is therefore related to dynamic isotropic percolation.

A refractory mechanism as the basis for the regulation of the spatio-temporal properties of retinal waves was first suggested in a model by Feller et al. (1997) and recently confirmed experimentally (Zheng et al., 2006). As in the present study, Feller et al. (1997) concluded that wave trajectories depend on the density of nonrefractory amacrine cells, which is determined by their activation history due to previous waves. This earlier model, however, required an additional filtering of amacrine cell activity through spatio-temporal integration and thresholding in RGCs to obtain compact waves. Here, we show that, in line with recent experimental and theoretical results (Zheng et al., 2006; Godfrey and Swindale, 2007), the properties of retinal waves are already present at the level of the SAC network. In addition, the model by Feller et al. (1997) required a certain degree of variability in the decay times of the refractory mechanism to prevent synchronization (Butts et al., 1999). A similar solution was recently suggested in a modeling study by Godfrey and Swindale (2007), where a highly nonlinear mechanism was used to amplify difference in refractory times between cells in the center and near the borders of waves. In this lattice model, the resulting network exhibits chaotic behavior, which may be a consequence of these severe nonlinearities.

Here, we reconcile and extend these previous approaches by suggesting a physiologically plausible mechanism for network desynchronization, which is based on differences between intrinsic bursting and depolarizations in SACs (Zheng et al., 2006), and allows us to provide a general theoretical framework to explain the origins of retinal waves. Given its generality, this principle might also apply to other development neural systems.

\section{References}

Abel HJ, Lee JC, Callaway JC, Foehring RC (2004) Relationships between intracellular calcium and after hyperpolarizations in neocortical pyramidal neurons. J Neurophysiol 91:324-335.

Bak P, Tang C, Wiesenfeld K (1987) Self-organized critically: an explanation of the 1/f noise. Phys Rev Lett 59:381-384.

Bansal A, Singer JH, Hwang BJ, Xu W, Beaudet A, Feller MB (2000) Mice lacking specific nicotinic acetylcholine receptor subunits exhibits dramatically altered spontaneous activity patterns and reveal a limited role for retinal waves in forming on and off circuits in the inner retina. J Neurosci 20:7672-7681.

Beggs JM, Plenz D (2003) Neuronal avalanches in neocortical circuits. J Neurosci 23:11167-11177.

Beggs JM, Plenz D (2004) Neuronal avalanches are diverse and precise activity patterns that are stable for many hours in cortical slice cultures. J Neurosci 24:5216-5229.

Buice MA, Cowan JD (2007) Field-theoretic approach to fluctuation effects in neural networks. Phys Rev E Stat Nonlin Soft Matter Phys 75:051919.

Butts DA, Feller MB, Shatz CJ, Rokhsar DS (1999) Retinal waves are governed by collective network properties. J Neurosci 19:3580-3593.

Cang J, Niell CM, Liu X, Pfeiffenberger C, Feldheim DA, Stryker MP (2008) Selective disruption of one cartesian axis of cortical maps and receptive 
fields by deficiency in ephrin-as and structured activity. Neuron 57:511-523.

Catsicas M, Bonness V, Becker D, Mobbs P (1998) Spontaneous Ca2 + transients and their transmission in the developing chick retina. Curr Biol $8: 283-286$.

Clauset A, Shalizi CR, Newman MEJ (2007) Power-law distributions in empirical data. arXiv/0706.1062.

Dickman R, Muñoz MA, Vespignani A, Zapperi S (2000) Paths to selforganized criticality. Braz J Phys 30:27-41.

Essam JW (1980) Percolation theory. Rep Prog Phys 43:833-912.

Eurich CW, Herrmann JM, Ernst UA (2002) Finite-size effects of avalanche dynamics. Phys Rev E Stat Nonlin Soft Matter Phys 66:066137.

Feller MB (1999) Spontaneous correlated activity in developing neural circuits. Neuron 22:653-656.

Feller MB, Wellis DP, Stellwagen D, Werblin FS, Shatz CJ (1996) Requirement for cholinergic synaptic transmission in the propagation of spontaneous retinal waves. Science 272:1182-1187.

Feller MB, Butts DA, Aaron HL, Rokhsar DS, Shatz CJ (1997) Dynamic processes shape spatiotemporal properties of retinal waves. Neuron 19:293-306.

Field DJ (1987) Relations between the statistics of natural images and the response properties of cortical cells. J Opt Soc Am A 4:2379-2394.

Firth SI, Wang CT, Feller MB (2005) Retinal waves: mechanisms and function in visual system development. Cell Calcium 37:425-432.

Gireesh ED, Plenz D (2008) Neuronal avalanches organize as nested thetaand beta/gamma- oscillations during development of cortical layer $2 / 3$. Proc Natl Acad Sci U S A 105:7576-7581.

Godfrey KB, Swindale NV (2007) Retinal wave behavior through activitydependent refractory periods. PLoS Comput Biol 3:e245.

Hopfield JJ, Herz AV (1995) Rapid local synchronization of action potentials: toward computation with coupled integrate-and-fire neurons. Proc Natl Acad Sci U S A 92:6655-6662.

Levina A, Herrmann JM, Geisel T (2007) Dynamical synapses causing selforganized criticality in neural networks. Nature Phys 3:857-860.

McLaughlin T, Torborg CL, Feller MB, O’Leary DD (2003) Retinotopic map refinement requires spontaneous retinal waves during a brief critical period of development. Neuron 40:1147-1160.

Mehta V, Sernagor E (2006) Early neural activity and dendritic growth in turtle retinal ganglion cells. Eur J Neurosci 24:773-786.

Moody WJ, Bosma MM (2005) Ion channel development, spontaneous activity, and activity-dependent development in nerve and muscle cells. Physiol Rev 85:883-941.

Morris C, Lecar H (1981) Voltage oscillations in the barnacle giant muscle fiber. Biophys J 35:193-213.

Muir-Robinson G, Hwang BJ, Feller MB (2002) Retinogeniculate axons undergo eye-specific segregation in the absence of eye-specific layers. J Neurosci 22:5259-5264.

Muñoz MA, Dickman R, Vespignani A, Zapperi S (1999) Avalanche and spreading exponents in systems with absorbing states. Phys Rev E Stat Nonlin Soft Matter Phys 59:6175-6179.

O’Donovan MJ (1999) The origin of spontaneous activity in developing networks of the vertebrate nervous system. Curr Opin Neurobiol 9:94-104.

Priesemann V, Wibral M, Munk M (2007) Detection of neuronal avalanches under incomplete sampling conditions in models of self- organized criticality and the macaque brain. Soc Neurosci Abstr 33:102.14/ZZ16.
Ribeiro TL, Copelli M (2008) Deterministic excitable media under poisson drive: Power law responses, spiral waves, and dynamic range. Phys Rev E Stat Nonlin Soft Matter Phys 77:051911.

Sernagor E, Grzywacz NM (1996) Influence of spontaneous activity and visual experience on developing retinal receptive fields. Curr Biol 6:1503-1508.

Sernagor E, Grzywacz NM (1999) Spontaneous activity in developing turtle retinal ganglion cells: pharmacological studies. J Neurosci 19:3874-3887.

Sernagor E, Eglen SJ, O'Donovan MJ (2000) Differential effects of acetylcholine and glutamate blockade on the spatiotemporal dynamics of retinal waves. J Neurosci 20:RC56(1-6).

Sernagor E, Eglen SJ, Wong RO (2001) Development of retinal ganglion cell structure and function. Prog Retin Eye Res 20:139-174.

Sernagor E, Young C, Eglen SJ (2003) Developmental modulation of retinal wave dynamics: shedding light on the GABA saga. J Neurosci 23:7621-7629.

Shah RD, Crair MC (2008) Retinocollicular synapse maturation and plasticity are regulated by correlated retinal waves. J Neurosci 28:292-303.

Stellwagen D, Shatz CJ, Feller MB (1999) Dynamics of retinal waves are controlled by cyclic AMP. Neuron 24:673-685.

Suding PN, Ziff RM (1999) Site percolation thresholds for archimedean lattices. Phys Rev E Stat Phys Plasmas Fluids Relat Interdiscip Topics 60:275-283.

Sun C, Warland DK, Ballesteros JM, van der List D, Chalupa LM (2008) Retinal waves in mice lacking the beta2 subunit of the nicotinic acetylcholine receptor. Proc Natl Acad Sci U S A 105:13638-13643.

Syed MM, Lee S, Zheng J, Zhou ZJ (2004) Stage-dependent dynamics and modulation of spontaneous waves in the developing rabbit retina. J Physiol 560:533-549.

Vespignani A, Zapperi S (1998) How self-organized criticality works: a unified mean-field picture. Phys Rev E Stat Nonlin Soft Matter Phys 57:6345-6362.

Wang CT, Blankenship AG, Anishchenko A, Elstrott J, Fikhman M, Nakanishi S, Feller MB (2007) $\mathrm{GABA}_{\mathrm{A}}$ receptor-mediated signaling alters the structure of spontaneous activity in the developing retina. J Neurosci 27:9130-9140.

Wong RO, Meister M, Shatz CJ (1993) Transient period of correlated bursting activity during development of the mammalian retina. Neuron 11:923-938.

Wong ROL (1999) Retinal waves and visual system development. Annu Rev Neurosci 22:29-47.

Yntema CL (1968) A series of stages in the embryonic development of chelydra serpentina. J Morphol 125:219-251.

Zapperi S, Bækgaard Lauritsen K, Stanley HE (1995) Self-organized branching processes: mean-field theory for avalanches. Phys Rev Lett 75:4071-4074.

Zheng J, Lee S, Zhou ZJ (2006) A transient network of intrinsically bursting starburst cells underlies the generation of retinal waves. Nat Neurosci 9:363-371.

Zheng JJ, Lee S, Zhou ZJ (2004) A developmental switch in the excitability and function of the starburst network in the mammalian retina. Neuron 44:851-864.

Zhou ZJ, Zhao D (2000) Coordinated transitions in neurotransmitter systems for the initiation and propagation of spontaneous retinal waves. J Neurosci 20:6570-6577. 